\title{
Awareness of an Primitive Art for Treating Otological Diseases in Present Covid-19 Scenario: Need of the Hour
}

\author{
Sweta Soni $^{1} \cdot$ Nisha shrivastava $^{1} \cdot$ Bhartendu Bharti $^{2}\left(\right.$ Vijay $^{-}$kumar $^{1} \cdot$ \\ Rudra prakash $^{1} \cdot$ Swati Suneha $^{1} \cdot$ Saraj K. Singh $^{3} \cdot$ Kranti Bhavana $^{1}$
}

Received: 2 October 2020/Accepted: 16 October 2020/Published online: 23 October 2020

(C) Association of Otolaryngologists of India 2020

\begin{abstract}
In current scenario of Covid-19 pandemic spread of virus via aerosol generating procedures has become a special concern in otorhinolaryngology community. Motive of this study is to spread awareness of an ancient forgotten method of performing mastoidectomy through which risk of virus infection can be greatly reduced among otologic surgeons. Retrospective Observational study. Three patients of chronic otitis media with complication were operatively intervened with combined approach of otorhinolaryngology and neurosurgeons. Mastoidectomies were performed without drilling. Instruments used were small, medium and large size gouge, chisel and hammer, curettes, kerrison punches and other microscopic ear instruments. All three patients recovered well without any residual disease or cavity problems. Referring ancient practices like above in this COVID era may prove an important tool in addressing surgical urgencies while combating transmission risks at the same time. One should be vigilant and versatile in surgical techniques in order to serve the needy and save the saviours simultaneously.
\end{abstract}

Keywords Mastoidectomies in Covid era .

Primitive mastoidectomy .

Chisel and hammer mastoid surgery

Bhartendu Bharti

bhartendubharti@gmail.com

1 Department of ENT, All India Institute of Medical Sciences, Patna, India

2 Department of Otorhinolaryngology and Head and Neck Surgery, Department of ENT, All India Institute of Medical Sciences, Patna, India

3 Department of Neurosurgery, All India Institute of Medical Sciences, Patna, India

\section{Introduction}

Covid-19 pandemic has changed our modern day medical practice in numerous different ways. Challenges are there not only in managing patients but also in preventing transmission of this disease to health care professionals. This has led to several modifications in approach to patients and their surgical management. Mastoid surgery being a significant aerosol generating procedure, owing to drilling and continuous irrigation, requires absolute indications and should be limited to emergency procedures only during the pandemic.

Using gouge and hammer to perform mastoid exploration causes much less aerosol generation as the bone chips are larger and heavier pieces of bone that tends to stay around the surgical field without much spread in and around the area. We present here series of three cases of emergency modified radical mastoidectomy done using a gouge and hammer instead of a drill. In this study we intend to demonstrate an ancient method of performing mastoid procedures which becomes the need of the hour by decreasing the amount of aerosol generation to minimum and make the procedure safer and secure for all Operation room personnel.

\section{Case Series Description}

- A 8 year old child who presented in our emergency in very debilitated condition having GCS score of 9/15. Evaluated by departments of ENT, neurosurgery and paediatrics based on clinic-radiological features he was diagnosed to have left sided brain abscess of otologic foci of infection. NCCT Brain suggested multiple cerebral abscess in left parietal temporal and occipital 
lobe with focal erosion of tegmen tympani (Fig. 1). MRI brain revealed opacities in middle ear and mastoid with erosion of ossicles, Posterior EAC and tegmen tympani, with huge focal lesion in brain parenchyma. An emergency burr hole drainage of abscess along with mastoid exploration was planned in single sitting. Though a COVID RT-PCR test sample was taken but we didn't waited for report to come and took patient for operative intervention combining both neurosurgical and otorhinological teams. Hence, we preferred taking all precautions including PPE for all OR personnel and safety measures for overall OR environment. Burr hole was done using minimal drilling and pus was evacuated. Gouge and hammer was used to open the mastoid at the Mac Ewan's triangle. Mastoid cavity was slowly enlarged by chiselling out bone chips and clearing it with intermittent saline washes. Canal wall was eroded by cholesteatoma and was further removed to achieve a "canal wall down" mastoid cavity. Cholesteatoma sac was seen in middle ear and was removed completely, incus was eroded and malleus handle and stapes footplate was preserved.

- Focal erosion of sigmoid plate was seen with exposure of dura (Fig. 2). Cavity was cleaned thoroughly with saline washes, a wide conchomeatoplasty was done and temporalis fascia graft was placed under handle of malleus. All of this was done under the microscope with excellent visibility. The child was shifted to paediatric

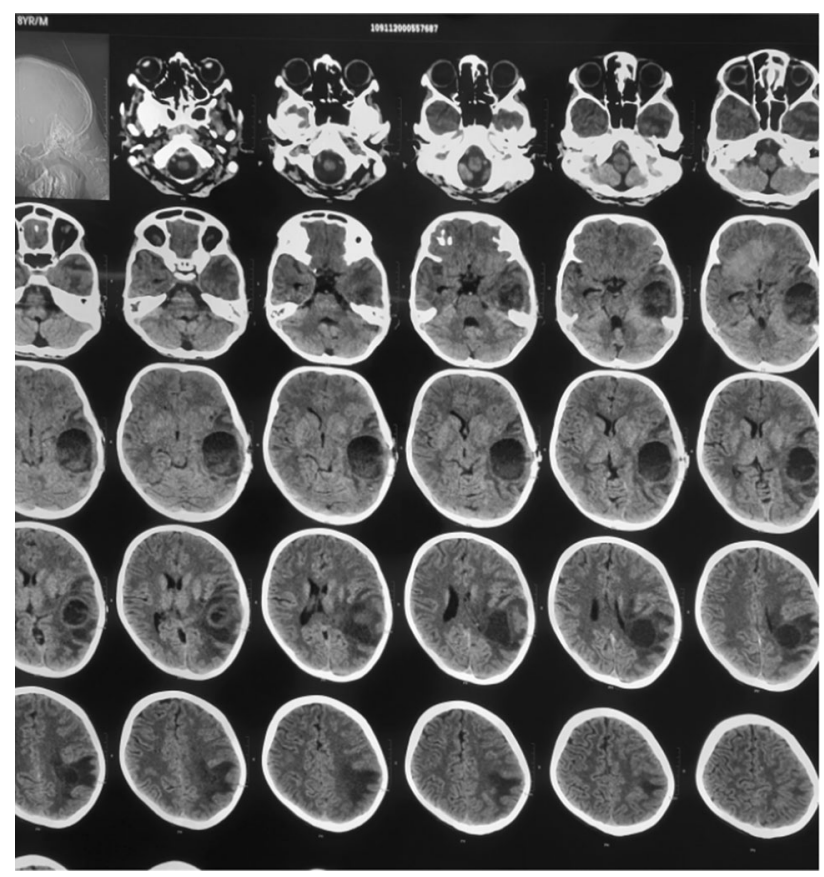

Fig. 1 MRI brain revealing opacities in middle ear and mastoid with erosion of ossicles, Posterior EAC and tegmen tympani, with huge focal lesion in brain parenchyma

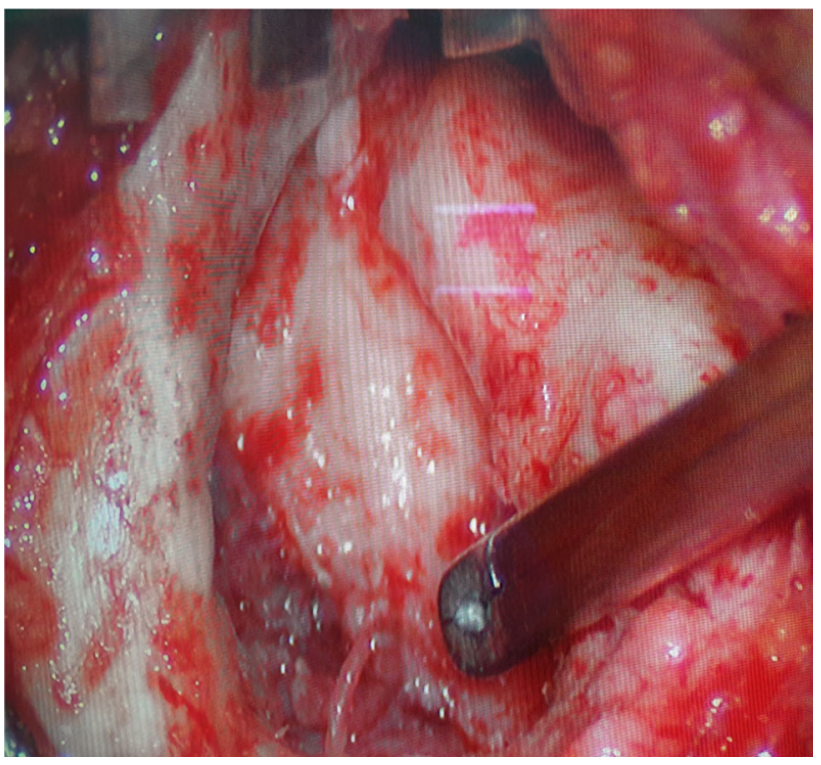

Fig. 2 Focal erosion of sigmoid plate was seen with exposure of dura

ICU considering his serious condition where he stayed for $72 \mathrm{~h}$ under strict monitoring. After that he was shifted to ward for further management. He recovered well and was discharged after 3 weeks of hospital stay with no residual neurological deficit.

- Two other patient one a18 year old boy and another an 16 year old boy came with complaints of headache, vomiting, fever and ear discharge (Fig. 3). A clinicoradiological diagnosis of bilateral squamosal otitis media with intracranial temporal brain abscess was made (Figs. 4 and 5). After a negative COVID test report in both cases and ensuring all safety precautions as described above, a burr hole was done and pus was evacuated. Gouge hammer was used to perform a

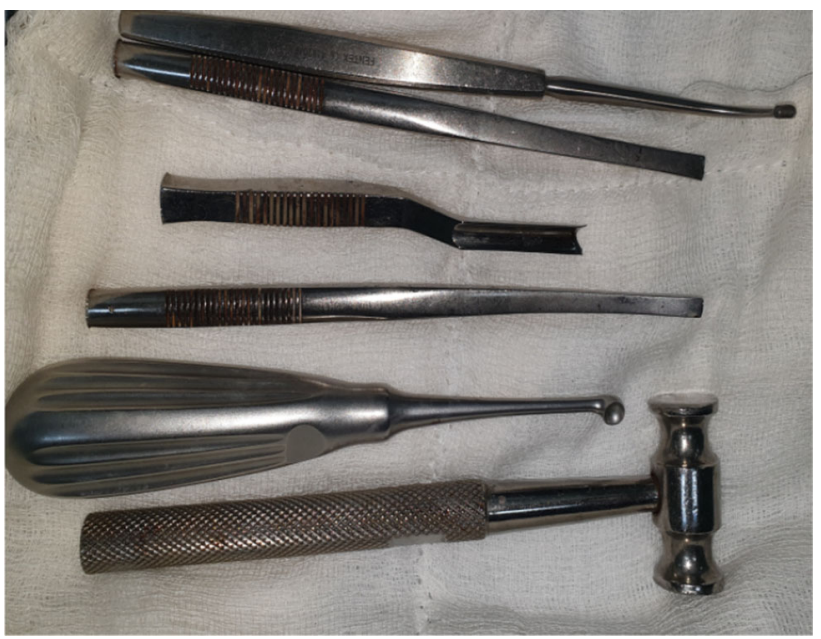

Fig. 3 Instruments used for mastoidectomy instead of drills 


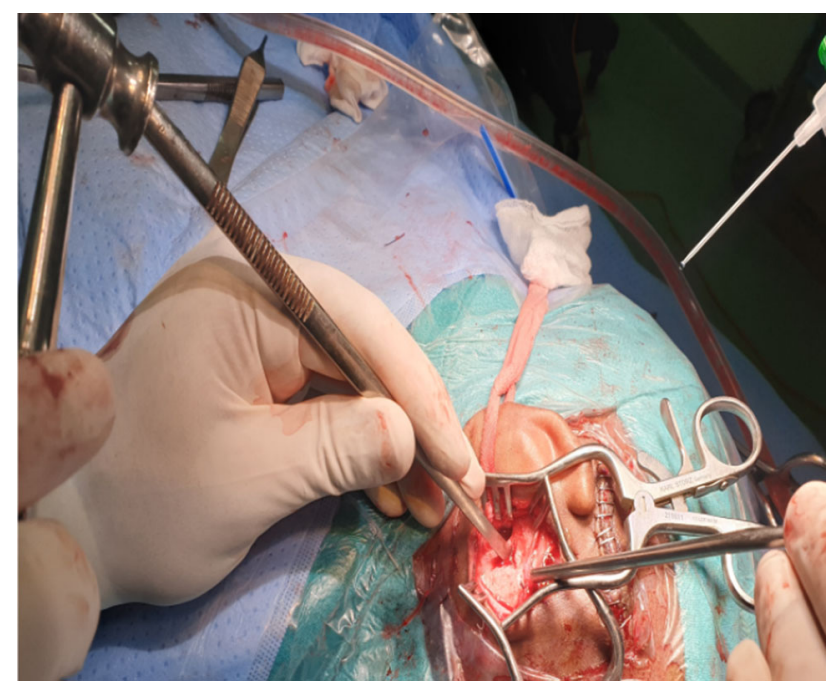

Fig. 4 Chiselling out bone over McEwan's triangle

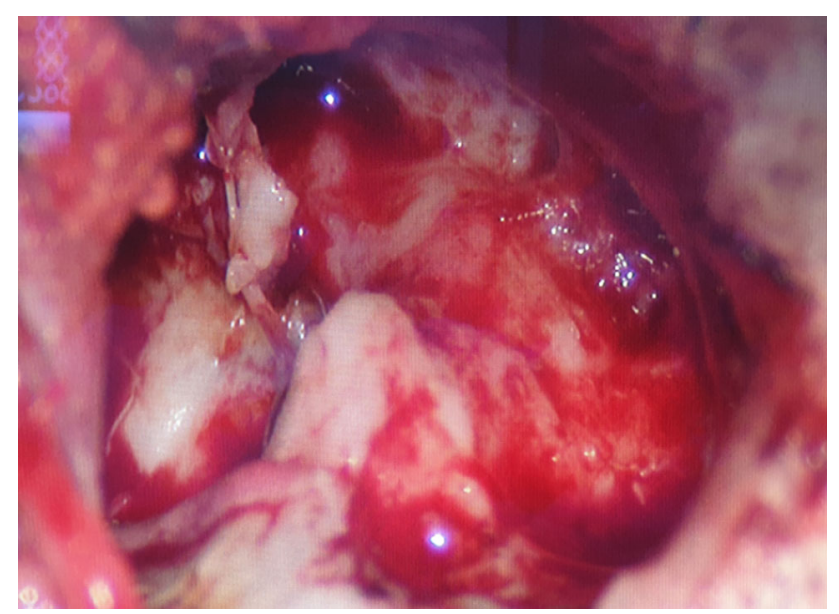

Fig. 5 Left canal wall down mastoid cavity with erosion of dural plate

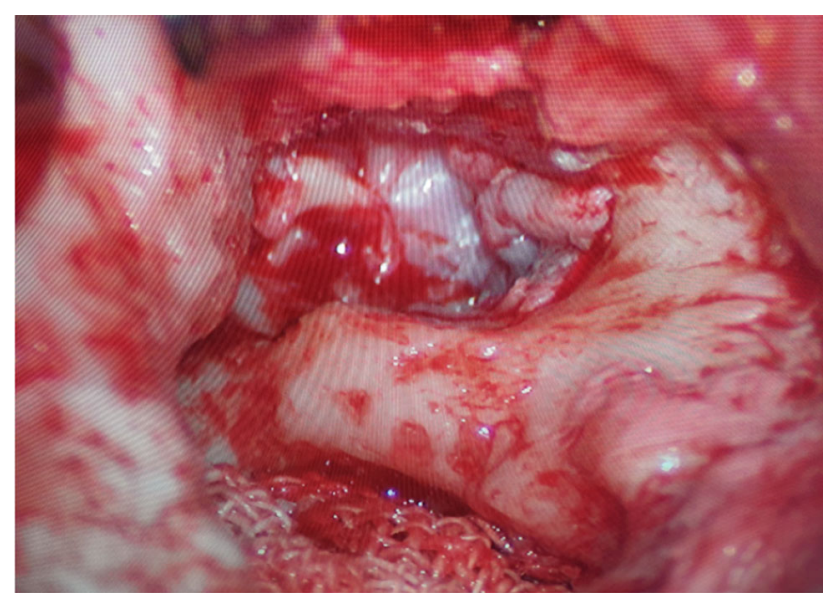

Fig. 6 Right canal wall down mastoid cavity with cholesteatoma in middle ear modified radical mastoidectomy (Fig. 6). Postoperative period was uneventful.

\section{Discussion}

Drills have multiple advantages but is dangerous in COVID times as it can be a source of "high speed aerosols." These aerosols can be a source of serious infections to the surgeons and other OR personnel. We used gouge and hammer to perform mastoid exploration in three patients thus avoiding "high speed aerosols" generation and ensuring adequate disease clearance.

Surgery involving the middle ear and mastoid, owing to their continuity with the upper aerodigestive tract, should be considered high risk for COVID-19 transmission. Whenever possible, mastoidectomy may need to be deferred, but if the surgery is required, enhanced PPE usage is advised and use of high-speed drills are to be avoided. Using hammer and gouge definitely decreases the amount of aerosol generation and hence decreasing risk of transmission manifolds.

Petit, in 1736, described using a chisel to remove the bone along an existing fistula. In 1873, Schwartze defined the concept of a standard surgical procedure for mastoidectomy, recommending the use of a chisel and hammer to remove the bone in such a way that the antrum could be properly inspected and efficiently drained [1]. Bondy described the use of curettes and gouges in surgery [2]. As stated by Dhruv et al. in their study mastoidectomy results in gross contamination of surgical site at 1 and $3 \mathrm{ft}$ in all directions [3]. Mastoidectomy without a barrier, even when a second suction is added, generated substantial 1-10 $\mu \mathrm{m}$ aerosols [4]. Drilling without a microscope dispersed fluorescent particles $360^{\circ}$, with the areas of highest density in quadrants near the surgeon and close to the surgical site [5]. Appropriate protective gear like an N-95 mask, goggles with face shield should be worn to reduce risk of transmission. Using gouge and chisels instead of drilling significantly reduces the amount of splatter and larger particle size limits area and directionality of spread. In experienced hands gouge hammer mastoidectomy has almost similar outcomes as those with motor drilling.

\section{Conclusion}

Pandemics have always mandated crucial reformatting and necessary adaptations to ensure survival. Referring ancient practices like above in this COVID era may prove an important tool in addressing surgical urgencies while combating transmission risks at the same time. This 
pandemic have forced us to think about training programmes during residency and to continue the exposure of old methods also to them during there tenure. One should be vigilant and versatile in surgical techniques in order to serve the needy and save the saviours simultaneously.

We never know when an old untouched stone might lead our path.

Funding No funding from any source.

Data Availability Available in Hospital information system.

\section{Compliance with Ethical Standards}

Conflict of interest There was no conflict of interest of any author.

Ethical Approval Ethical approval was waived by the local Ethics Committee of institution, In view of the retrospective nature of the study and all the procedures being Performed was part of the routine care.

Informed Consent Informed consent was taken from patient and/or relatives for data publication.

\section{References}

1. Mudry A (2009) History of instruments used for mastoidectomy. J LaryngolOtol 123(6):583-589. https://doi.org/10.1017/S0022215109004484

2. Bento RF, Fonseca AC (2013) A brief history of mastoidectomy. Int Arch Otorhinolaryngol 17(2):168-178. https://doi.org/10.7162/S1809-97772013000200009

3. Sharma D, Rubel KE, Ye MJ, Campiti VJ, Carroll AE, Ting JY, Illing EA, Burgin SJ (2020) Cadaveric simulation of otologic procedures: an analysis of droplet splatter patterns during the COVID-19 pandemic. Otolaryngol Head Neck Surg. https://doi.org/10.1177/0194599820930245

4. Chari DA, Workman AD, Chen JX et al (2020) Aerosol dispersion during mastoidectomy and custom mitigation strategies for otologic surgery in the COVID-19 era. Otolaryngol Head Neck Surg. https://doi.org/10.1177/0194599820941835

5. Chen JX, Workman AD, Chari DA et al (2020) Demonstration and mitigation of aerosol and particle dispersion during mastoidectomy relevant to the COVID-19 era. OtolNeurotol. https://doi.org/10.1097/MAO.0000000000002765

Publisher's Note Springer Nature remains neutral with regard to jurisdictional claims in published maps and institutional affiliations. 